Proceedings of the

International Geometry Center

Vol. 13, no. 1 (2020) pp. 35-48

\title{
Geodesic mappings of compact quasi-Einstein spaces, I
}

\author{
V. Kiosak, A. Savchenko, G. Kovalova
}

\begin{abstract}
The paper treats a particular type of pseudo-Riemannian spaces, namely quasi-Einstein spaces with gradient defining vector. These spaces are a generalization of well-known Einstein spaces. There are three types of these spaces that admit locally geodesic mappings. Authors proved a "theorem of disappearance" for compact quasi-Einstein spaces of main type.
\end{abstract}

Анотація. В роботі досліджується спеціальний тип псевдоріманових просторів - майже ейнштейнові простори з градієнтним задаючим вектором. Ці простори узагальнюють відомі простори Ейнштейна, які характеризуються тим, що тензор Ейнштейна дорівнює нулю. В майже ейштейнових просторах тензор Ейнштейна відхиляється від нуля на деяку величину, яку називають дефектом тензора Ейнштейна. Якщо дефект тензора Ейнштейна це деякий простий бівектор, то простори називають майже ейнштейновими з градієнтним задаючим вектором.

Основним методом моделювання фізичних та інших процесів, що характеризуються за допомогою псевдоріманових просторів, є їх відображення на той чи інший спеціальний тип просторів. Багатьма авторами розглядались конформні та геодезичні відображення майже ейнштейнових просторів.

В цій роботі вивчаємо геодезичні відображення майже ейнштейнових просторів з градієнтним задаючим вектором за допомогою лінійної форми основних рівнянь теорії геодезичних відображень. Через різного типу обмеження алгебраїчного та диференціального характеру майже ейнштейнові простори, які допускають нетривіальні геодезичні відображення, розбиті на три типи: основний, спеціальний та особливий.

Використовуючи поняття геодезичних точок, приходимо до розгляду геодезичних відображень "в цілому". Для отримання результатів в компактних ріманових просторах застосовуємо теорему Хопфа-Бохнера в вигляді, який запропонувала О. М. Синюкова. Теореми про не існування "в цілому" певних типів просторів називають "теоремами зникнення". Для компактних майже ейнштейнових просторів основного типу доведено "теореми зникнення". А саме, компактний майже ейнштейнів простір

$U D C 514.765 .1+512.813 .4$

Keywords: pseudo-Riemannian spaces; quasi-Einstein spaces; geodesic mapping DOI: http://dx.doi.org/10.15673/tmgc.v13i1.1711 
основного типу з додатно визначеною метрикою та додатною скалярною кривиною не допускає нетривіальних геодезичних відображень "в цілому".

Отримані результати дозволяють ефективно продовжити дослідження, вивчити особливий та спеціальний типи майже ейнштейнових просторів, а також, використовуючи відомі методи зробити висновки про геодезичні відображення повних псевдоріманових просторів. Дослідження ведуться в тензорній формі в класі достатньо гладких функцій.

\section{INTRODUCTION}

We will study a pseudo-Riemannian space $V_{n},(n>2)$, with a metric tensor $g_{i j}$ and construct an Einstein tensor in this space. Such a tensor is defined by a well-known expression:

$$
E_{i j}:=R_{i j}-\frac{R}{n} g_{i j}
$$

where $R_{i j}:=R_{i j \alpha}^{\alpha}$ is the Ricci tensor, $R=R_{\alpha \beta} g^{\alpha \beta}$ is the scalar curvature, and $R_{i j k}^{h}$ is the Riemannian tensor.

The defect of Einstein tensor [6] is a tensor $D_{i j}$, defined by the equation

$$
E_{i j}-D_{i j}=0 .
$$

When selecting a special type of tensor $D_{i j}$, one can select a particular type of special pseudo-Riemannian spaces. For example, if $D_{i j}$ is a linear combination of the metric tensor and the covariant derivative of a certain vector, then taking into account coefficients of this combination, one can obtain $\varphi($ Ric $)$-spaces or Ricci solitons [3, 4].

When $D_{i j}$ is represented by a simple bivector, called determining, then the space is quasi-Einstein [8].

Mapping is a main way for modeling of the above-mentioned spaces. We conducted a research aimed at conformal and geodesic mappings of pseudoRiemannian spaces with various types of deformation tensor of Einstein tensor $[2,5,9]$.

This work treats geodesic mappings of quasi-Einstein spaces with gradient defining vector. These spaces are subdivided into three types: main, particular and special.

The obtained local results are applied to the study of compact quasiEinstein spaces of main type "in the large".

\section{BASiC EQUations of GEODESIC MAPPINGS THEORY}

Bijection of points of pseudo-Riemannian spaces $V_{n}$ with a metric tensor $g_{i j}$ and $\bar{V}_{n}$ with a metric tensor $\bar{g}_{i j}$ is called a geodesic mapping whenever each geodesic line $V_{n}$ is transformed into a geodesic line $\bar{V}_{n}$. 
Pseudo-Riemannian spaces $V_{n}$ and $\bar{V}_{n}$ admitting a geodesic mapping between them are called spaces in geodesic correspondence or belonging to the same geodesic class.

The following identity [13] gives a necessary and sufficient condition for defining a bijective geodesic mapping between pseudo-Riemannian spaces $V_{n}$ and $\bar{V}_{n}$ :

$$
\bar{\Gamma}_{i j}^{h}=\Gamma_{i j}^{h}+\varphi_{i} \delta_{j}^{h}+\varphi_{j} \delta_{i}^{h}
$$

or otherwise, taking into account a covariant constancy of a metric tensor,

$$
\bar{g}_{i j, k}=2 \varphi_{k} \bar{g}_{i j}+\varphi_{i} \bar{g}_{j k}+\varphi_{j} \bar{g}_{i k},
$$

here $\varphi_{i}$ is a certain (necessarily gradient) vector, $\Gamma_{i j}^{h}$ and $\bar{\Gamma}_{i j}^{h}$ are the Christoffel symbols of $V_{n}$ and $\bar{V}_{n}$ respectively, $\delta_{i}^{h}$ is a Kronecker symbols, and comma "," is a sign of covariant derivatives in respect to the connection of $V_{n}$.

Equations (1.1) and (1.2) are equivalent, and they are necessary and sufficient existence of a bijective geodesic correspondence between pseudoRiemannian spaces $V_{n}$ and $\bar{V}_{n}$.

The following relations a necessary for an existence of a geodesic mapping:

$$
\begin{gathered}
\bar{R}_{i j k}^{h}=R_{i j k}^{h}+\varphi_{i j} \delta_{k}^{h}-\varphi_{i k} \delta_{j}^{h}, \\
\bar{R}_{i j}=R_{i j}+(n-1) \varphi_{i j},
\end{gathered}
$$

where $\varphi_{i j}=\varphi_{i, j}-\varphi_{i} \varphi_{j}$, and $R_{i j k}^{h}$ and $R_{i j k}$ are respectively Riemannian and Ricci tensors.

A geodesic mapping distinct from homothety is called non-trivial.

A pseudo-Riemannian space $V_{n}$ admits a non-trivial geodesic map if and only if it contains a solution of a system of differential equations in respect to the tensor $a_{i j}=a_{j i} \neq c g_{i j}$ and the vector $\lambda_{i}=\lambda_{, i} \neq 0$. This system is called a linear form of main equations.

Linear form of main equations for geodesic mappings theory can be written as follows [13]:

$$
\begin{gathered}
a_{i j, k}=\lambda_{i} g_{j k}+\lambda_{j} g_{i k}, \\
n \lambda_{i, j}=\mu g_{i j}+a_{\alpha i} R_{j}^{\alpha}-a_{\alpha \beta} R_{. i j}^{\alpha{ }^{\beta}},
\end{gathered}
$$

where $\mu=\lambda_{\alpha, \beta} g^{\alpha \beta}, R_{j}^{i}=R_{\alpha j} g^{\alpha i}$, and $R^{h{ }_{i j}{ }^{k}}=R_{i j \alpha}^{h} g^{\alpha}$. ${ }^{k}$

It follows from the latter equations that

$$
(n-1) \mu_{, i}=2(n+1) \lambda_{\alpha} R_{i}^{\alpha}+a_{\alpha \beta}\left(2 R_{\left.. i,{ }_{.}{ }^{\beta}-R_{, i}^{\alpha \beta}\right) .}\right.
$$

Solutions (1.2) and (1.3) are related by the following identity:

$$
a_{i j}=e^{2 \varphi} \bar{g}^{\alpha \beta} g_{\alpha i} g_{\beta j},
$$




$$
\lambda_{i}=-e^{2 \varphi} \bar{g}^{\alpha \beta} g_{\alpha i} g_{\beta}
$$

The system of equations (1.3), (1.4), and (1.5) gives a possibility to answer the question whether a certain pseudo-Riemannian space $V_{n}$ admits a geodesic mapping onto a pseudo-Riemannian space $\bar{V}_{n}$. This problem is reduced to finding the integrability conditions of these equations and their differential extensions. Such a system is called a system of main equations of theory of geodesic mappings $[10,7,12]$.

\section{BASIC EQUATIONS OF THEORY OF GEODESIC MAPPINGS FOR QUASI-EINSTEIN SPACES}

We will study geodesic mappings of quasi-Einstein spaces, namely pseudoRiemannian spaces $V_{n}(n>2)$, satisfying the following conditions:

$$
R_{i j}=\frac{R}{n} g_{i j}+U_{i} U_{j}
$$

where $U_{i}$ is defined as gradient vector, or otherwise

$$
U_{i}=U_{, i}=\partial_{i} U
$$

The definition implies that the vector $U_{i}$ is necessarily isotropic, [8].

Differentiating (2.1) we get

$$
R_{i j . k}=\frac{1}{n} R_{, k} g_{i j}+U_{i, k} U_{j}+U_{i} U_{j, k} .
$$

Contracting indices transforms it into

$$
R_{i \alpha,}^{\alpha}=\frac{1}{n} R_{, i}+U_{\alpha,}^{\alpha} U_{i}
$$

or

$$
R_{, i}=\frac{2 n}{n-1} U_{\alpha,}^{\alpha} U_{i}
$$

Then (2.2) can be written as follows:

$$
R_{i j . k}=\frac{2}{n-1} U_{\alpha,}^{\alpha} U_{k} g_{i j}+U_{i, k} U_{j}+U_{i} U_{j, k} .
$$

Symmetrizing (1.4) we will see that

$$
a_{\alpha l} R_{h}^{\alpha}-a_{\alpha k} R_{l}^{\alpha}=0 .
$$

Hence, taking into account (2.1), we can rewrite (2.4) as follows:

$$
U_{l} U^{\alpha} a_{\alpha i}=U_{i} a_{\alpha l} U^{\alpha}
$$

The latter implies that

$$
U^{\alpha} a_{\alpha i}=\rho U_{i}
$$

where $\rho:=a_{\alpha \beta} U^{\alpha} \xi^{\beta}$ and $\xi^{i}$ is a certain vector chosen so that $U_{\alpha} \xi^{\alpha}=1$. Thus, we have established the following

Theorem 2.1. If a quasi-Einstein space $V_{n}$ admits a non-trivial geodesic mapping, then the vector $U_{i}$ is an eigenvector of the matrix of tensor $a_{i j}$. 
Let us prove the following

Theorem 2.2. If a quasi-Einstein space $V_{n}$ admits a non-trivial geodesic mappings, then the vectors $U_{i}$ and $\lambda_{i}$ are orthogonal each to other, namely

$$
U^{\alpha} \lambda_{\alpha}=0
$$

Proof. Differentiating (2.5) and taking into account (1.3) we get

$$
U^{\alpha}{ }_{, j} a_{\alpha i}+U^{\alpha} \lambda_{\alpha} g_{i j}+\lambda_{i} U_{j}=\rho_{, j} U_{i}+\rho U_{i, j}
$$

Multiplying (2.7) by isotropic vector $U_{i}$ and contracting indices we obtain

$$
2 U^{\alpha} \lambda_{\alpha} U_{i}=0 \text {. }
$$

Since the vector $U_{i}$ never is zero, the theorem is proved.

Taking into account the latter statement, one can rewrite (2.7) as follows

$$
U_{, j}^{\alpha} a_{\alpha i}=\rho, j U_{i}+\rho U_{i, j}-\lambda_{i} U_{j} .
$$

Moreover, multiplying $(2.8)$ by $U_{j}$ and $a_{k}^{\alpha}$, we get the following

Lemma 2.3. If a quasi-Einstein space admits non-trivial geodesic mappings, then the following condition is true

$$
\rho_{\alpha} U^{\alpha}=0, \quad \rho_{\alpha} a_{i}^{\alpha}-\rho \rho_{i}=\stackrel{1}{c} U_{i},
$$

where $\rho_{i}=\rho_{, i}=\partial_{i} \rho, \stackrel{1}{c}=\left(a_{\beta}^{\alpha} \rho_{\alpha}-\rho \rho_{\beta}\right) \xi^{\beta}$, and $U_{\alpha} \xi^{\alpha}=1$.

We will now prove the following

Theorem 2.4. If a quasi-Einstein space admits non-trivial geodesic mappings, then the vector $\lambda_{i}$ satisfies the following condition:

$$
\lambda^{\alpha} a_{\alpha i}=\tau U_{i}+\stackrel{1}{\tau} \lambda_{i},
$$

where $\tau$ is a certain invariant chosen in such a way that

$$
\tau:=2(n-1) A_{\alpha \beta} \lambda^{\alpha} \xi^{\beta}, \quad \quad \frac{1}{\tau}:=-\frac{\rho-a}{n-1},
$$

and $\xi^{i}$ is a vector that complies to condition $U_{\alpha} \xi^{\alpha}=1$.

Proof. By differentiating

$$
a_{\alpha i} R_{j k l}^{\alpha}+a_{\alpha j} R_{i k l}^{\alpha}=\lambda_{l i} g_{j k}+\lambda_{l j} g_{i k}-\lambda_{k j} g_{i l}-\lambda_{k i} g_{j l},
$$

where $\lambda_{i j}=\lambda_{i, j}$ and taking to account (1.3), we obtain

$$
\begin{array}{r}
\lambda_{\alpha} R_{j k l}^{\alpha} g_{i m}+\lambda_{i} R_{m j k l}+\lambda_{\alpha} R_{i k l}^{\alpha} g_{j m}+\lambda_{j} R_{m i k l}+a_{\alpha i} R_{j k l, m}^{\alpha}+a_{\alpha j} R_{i k l, m}^{\alpha}= \\
=\lambda_{l i, m} g_{j k}+\lambda_{l j, m} g_{i k}-\lambda_{k i, m} g_{j l}-\lambda_{k j, m} g_{i l} .
\end{array}
$$


Contracting indices in the latter expression with respect to $l$ and $m$, we can write down the expression

$$
\begin{aligned}
\lambda_{\alpha} R_{j k i}^{\alpha}+\lambda_{\alpha} R_{i k j}^{\alpha}+\lambda_{i} R_{j k}+\lambda_{j} R_{i k} & +a_{i}^{\alpha} R_{k j \alpha, \beta}^{\beta}+a_{j}^{\alpha} R_{k i \alpha, \beta}^{\beta}= \\
& =\lambda_{\alpha i,}{ }^{\alpha} g_{j k}+\lambda_{\alpha j}{ }^{\alpha}{ }^{\alpha} g_{i k}-\lambda_{k i, j}-\lambda_{k j, i} .
\end{aligned}
$$

Taking into account $R_{i j k, \alpha}^{\alpha}=R_{i j, k}-R_{i k, j}$ and (2.1), we get

$$
\begin{aligned}
& \lambda_{\alpha} R_{j k i}^{\alpha}+\lambda_{\alpha} R_{i k j}^{\alpha}+\lambda_{i} R_{j k}+\lambda_{j} R_{i k}+ \\
& \quad+U_{j}\left(\rho_{k} U_{i}+\rho U_{i, k}-\lambda_{i} U_{k}+\frac{2}{n-1} U_{\alpha}^{\alpha}, \rho g_{k i}-\frac{2}{n-1} U_{\alpha,}{ }^{\alpha} a_{i k}\right)- \\
& \quad-\rho U_{i} U_{k, j}+U_{i}\left(\rho_{k} U_{j}+\rho U_{j, k}-\lambda_{j} U_{k}+\frac{2}{n-1} U_{\alpha,}{ }^{\alpha} \rho g_{k j}-\frac{2}{n-1} U_{\alpha,}{ }^{\alpha} a_{j k}\right)- \\
& \quad-\rho U_{j} U_{k, i}=\lambda_{\alpha i,}{ }^{\alpha} g_{j k}+\lambda_{\alpha j,}{ }^{\alpha} g_{i k}-\lambda_{k i, j}-\lambda_{k j, i} .
\end{aligned}
$$

Hence

$$
\begin{aligned}
\lambda_{\alpha} R_{j k i}^{\alpha}+ & \lambda_{\alpha} R_{i k j}^{\alpha}+\lambda_{i} R_{j k}+\lambda_{j} R_{i k}+ \\
& +U_{j}\left(\rho_{k} U_{i}-\lambda_{i} U_{k}+\frac{2}{n-1} U_{\alpha}^{\alpha}, \rho g_{k i}-\frac{2}{n-1} U_{\alpha}{ }^{\alpha}, a_{i k}\right)+ \\
& +U_{i}\left(\rho_{k} U_{j}-\lambda_{j} U_{k}+\frac{2}{n-1} U_{\alpha}{ }^{\alpha}, \rho g_{k j}-\frac{2}{n-1} U_{\alpha}{ }^{\alpha}, a_{j k}\right)= \\
& =\lambda_{\alpha i,}{ }^{\alpha} g_{j k}+\lambda_{\alpha j}{ }^{\alpha}{ } g_{i k}-\lambda_{k i, j}-\lambda_{k j, i} .
\end{aligned}
$$

Alternating the latter by $j, k$, we get the equation

$$
\begin{aligned}
4 \lambda_{\alpha} R_{i k j}^{\alpha}=\left(\lambda_{\alpha j,}{ }^{\alpha},\right. & \left.-\frac{R}{n} \lambda_{j}-\frac{2}{n-1} U_{\alpha}{ }^{\alpha} U_{j}\right) g_{i k}- \\
& \quad\left(\lambda_{\alpha k,}{ }^{\alpha}-\frac{R}{n} \lambda_{k}-\frac{2}{n-1} U_{\alpha}{ }^{\alpha} U_{k}\right) g_{i j}+ \\
& \quad+2 U_{i}\left(U_{k} \rho_{j}-U_{j} \rho_{k}\right)+\frac{2}{n-1} U_{j} a_{i k}-\frac{2}{n-1} U_{k} a_{i j} .
\end{aligned}
$$

Contraction transforms it into

$$
\lambda_{\alpha j,}{ }^{\alpha}-\frac{R}{n} \lambda_{j}-\frac{2}{n-1} U_{\alpha,}{ }^{\alpha} U_{j}=\frac{4 R}{n(n-1)} \lambda_{j}+\frac{2}{n-1}\left(\frac{\rho-a}{n-1}\right) U_{j} .
$$

The latter means that

$$
\begin{aligned}
\lambda_{\alpha} R_{i k j}^{\alpha}=\frac{1}{2}\left(U_{i} U_{k} \rho_{j}-U_{j} U_{i} \rho_{k}\right)+ & U_{k} A_{i j}-U_{j} A_{i k}+ \\
& +\frac{R}{n(n-1)}\left(\lambda_{j} g_{i k}-\lambda_{k} g_{j i}\right),
\end{aligned}
$$

here

$$
A_{i j}=\frac{1}{2(n-1)}\left(a_{i j}+\left(\frac{\rho-a}{n-1}\right) g_{j i}\right)
$$


Let us multiply $(2.11)$ by $\lambda^{i}$ and contract it with respect to $i$. Then the expression can be written:

$$
U_{k} \lambda^{\alpha} A_{\alpha j}-U_{j} \lambda^{\alpha} A_{\alpha k}=0 .
$$

Thus,

$$
\lambda^{\alpha} A_{\alpha i}=\frac{\tau}{2(n-1)} U_{i},
$$

where $\tau$ is a certain invariant, such that $\tau:=2(n-1) A_{\alpha \beta} \lambda^{\alpha} \xi^{\beta}$, and $\xi^{i}$ is a vector complying the condition $U_{\alpha} \xi^{\alpha}=1$.

Thus, (2.9) is true which proves Theorem 2.4.

Multiplying (2.10) by $\lambda^{l}$, contracting it with respect to $l$, and taking into account $(2.11)$, we obtain

$$
\begin{aligned}
& \frac{1}{2}\left(\rho_{\alpha} a_{i}^{\alpha} U_{k} U_{j}-\rho \rho_{j} U_{k} U_{i}\right)+a_{i}^{\alpha} A_{\alpha k} U_{j}-\rho U_{i} A_{k j}+ \\
& +\frac{R}{n(n-1)}\left(a_{i}^{\alpha} \lambda_{\alpha} g_{k j}-\lambda_{j} a_{i k}\right)+\frac{1}{2}\left(\rho_{\alpha} a_{j}^{\alpha} U_{k} U_{i}-\rho \rho_{i} U_{k} U_{j}\right)+ \\
& \quad+a_{j}^{\alpha} A_{\alpha k} U_{i}-\rho U_{i} A_{k j}+\frac{R}{n(n-1)}\left(\lambda_{\alpha} a_{j}^{\alpha} g_{k i}-\lambda_{i} a_{j k}\right)= \\
& \quad=\lambda^{\alpha} \lambda_{\alpha i} g_{j k}+\lambda^{\alpha} \lambda_{\alpha j} g_{i k}-\lambda_{k j} \lambda_{i}-\lambda_{k i} \lambda_{j} .
\end{aligned}
$$

Alternate the latter expressions by $j$ and $k$, then exchange positions of indexes $i \leftrightarrow k$, and add the result to (2.12). Then we will get

$$
\begin{aligned}
\frac{1}{2} \stackrel{1}{c} U_{i} U_{k} U_{j}+U_{i} & \left(a_{j}^{\alpha} A_{\alpha k}-\rho A_{k j}\right)+ \\
& +\frac{R}{n(n-1)}\left(\lambda_{\alpha} a_{i}^{\alpha} g_{k j}-\lambda_{i} a_{j k}\right)=\lambda^{\alpha} \lambda_{\alpha i} g_{j k}-\lambda_{k j} \lambda_{i} .
\end{aligned}
$$

Contracting this with $g^{j k}$, we obtain

$$
\lambda^{\alpha} \lambda_{\alpha i}-\frac{R}{n(n-1)} \lambda^{\alpha} a_{\alpha i}+\frac{\rho}{2(n-1)} \cdot\left(\frac{\rho-a}{n-1}\right) \cdot U_{i}=\mu \lambda_{i}+\stackrel{2}{\tau} U_{i},
$$

where

$$
\begin{gathered}
\mu=\frac{1}{n}\left(\lambda_{\alpha \beta}-\frac{R}{n(n-1)} a_{\alpha \beta}\right) g^{\alpha \beta}, \\
\stackrel{2}{\tau}=\frac{1}{2 n(n-1)}\left(a_{\beta}^{\alpha} a_{\alpha \gamma}+a_{\beta \gamma}\left(\frac{\rho-a}{n-1}-\rho\right)\right) g^{\beta \gamma}, \\
\lambda_{i}\left(\lambda_{k j}-\frac{R}{n(n-1)} a_{k j}-\mu g_{k j}\right)= \\
=U_{i}\left(\frac{1}{2(n-1)}\left(a_{j}^{\alpha} a_{\alpha k}+\left(\frac{\rho-a}{n-1}-\rho\right) a_{k j}-\stackrel{2}{\tau} g_{k j}\right)+\frac{1}{2} \stackrel{1}{c} U_{k} U_{j}\right) .
\end{gathered}
$$

Contracting the latter expression with a vector $\xi^{i}$ chosen in such a way that $\xi^{\alpha} U_{\alpha}=1$, we can write down the expression

$$
\frac{1}{2(n-1)}\left(a_{j}^{\alpha} a_{\alpha k}+\left(\frac{\rho-a}{n-1}-\rho\right) a_{k j}-\stackrel{2}{\tau} g_{k j}\right)+\frac{1}{2} \stackrel{1}{c} U_{k} U_{j}=
$$




$$
=v\left(\lambda_{k j}-\frac{R}{n(n-1)} a_{k j}-\mu g_{k j}\right),
$$

where $v=\lambda_{\alpha} \xi^{\alpha}$. Therefore

$$
\left(\lambda_{i}-v U_{i}\right)\left(\mu g_{k j}-\lambda_{k j}+\frac{R}{n(n-1)} a_{k j}\right)=0 .
$$

Thus, either

$$
\lambda_{i, j}=\mu g_{i j}+\frac{R}{n(n-1)} a_{i j}
$$

or

$$
\lambda_{i}-v U_{i}=0
$$

is true. So, we proved the following

Theorem 2.5. If a quasi-Einstein space admits non-trivial geodesic mappings, then it satisfies one of the conditions (2.14) or (2.15).

According to the latter statement, quasi-Einstein spaces can be subdivided into three types:

Main type: when (2.14) is true, while (2.15) is not true;

Particular type: when (2.15) is true but (2.14) is not;

Special type: when both equations (2.14) and (2.15) are true.

Further, we are going to treat different types of quasi-Einstein spaces consequently.

\section{QUASI-EINSTEIN SPACES OF THE MAIN TYPE}

In this section we will study quasi-Einstein spaces of the main type. These are pseudo-Riemannian spaces satisfying conditions (1.3), (2.1) and (2.14). By differentiating (2.14), we can obtain the following:

$$
\lambda_{i, j k}=\mu_{k} g_{i j}+\frac{R}{n(n-1)}\left(\lambda_{i} g_{j k}+\lambda_{j} g_{i k}\right)+\frac{1}{n(n-1)} R_{, k} a_{i j},
$$

where $\mu_{k}=\mu_{, k}=\partial_{k} \mu$.

Alternating and taking into account Ricci identity and (1.1) we get

$$
\begin{aligned}
\lambda_{\alpha} R_{i j k}^{\alpha}=\mu_{k} g_{i j}-\mu_{j} g_{i k}+\frac{R}{n(n-1)}( & \left.\lambda_{j} g_{i k}-\lambda_{k} g_{i j}\right)+ \\
& +\frac{1}{n(n-1)} \cdot \frac{2 n}{n-1} \cdot U_{\alpha}{ }^{\alpha}\left(U_{k} a_{i j}-U_{j} a_{i k}\right) .
\end{aligned}
$$

Contracting and taking into account (2.1) and (2.6), we obtain

$$
\frac{R}{n} \lambda_{k}=(n-1) \mu_{k}-\frac{R}{n} \lambda_{k}+\frac{2}{(n-1)^{2}} U_{\alpha}{ }^{\alpha}(a-\rho) U_{k},
$$

or

$$
\mu_{k}=\frac{2 R}{n(n-1)} \lambda_{k}-\frac{2 U_{\alpha,}^{\alpha}}{(n-1)^{2}} \stackrel{1}{\tau} U_{k}
$$

The following is true: 
Lemma 3.1. The system of equations (1.3), (2.14), (3.1) has a solution in quasi-Einstein spaces belonging to the main type.

Taking to account (3.1) one can write down integrability conditions for equations (1.3) and (2.14) in the following form:

$$
\begin{gathered}
a_{\alpha i} Y_{j k l}^{\alpha}+a_{\alpha j} Y_{i k l}^{\alpha}=0, \\
\lambda_{\alpha} Y_{i j k}^{\alpha}=\frac{2 U_{\alpha,}^{\alpha}}{(n-1)^{2}}\left(U_{k} a_{i j}-U_{j} a_{i k}-{ }^{1} U_{k} g_{i j}+{ }^{1} U_{j} g_{i k}\right),
\end{gathered}
$$

where

$$
Y_{i j k}^{\alpha}=R_{i j k}^{h}-\frac{R}{n(n-1)}\left(\delta_{k}^{h} g_{i j}-\delta_{j}^{h} g_{i k}\right)
$$

is a tensor of concircular curvature.

Multiplying (3.2) by $\lambda^{l}$ and taking to account (3.3), we get

$$
\begin{aligned}
\frac{2 U_{\alpha}{ }^{\alpha}}{(n-1)^{2}}\left(U _ { k } \left(\rho a_{i j}-a_{\alpha i} a_{j}^{\alpha}-\stackrel{1}{\tau} \rho g_{i j}\right.\right. & \left.+\stackrel{1}{\tau} a_{i j}\right)+ \\
& \left.+U_{j}\left(\rho a_{i k}-a_{\alpha i} a_{k}^{\alpha}-\frac{1}{\tau} \rho g_{i k}+\stackrel{1}{\tau} a_{i k}\right)\right)=0 .
\end{aligned}
$$

The latter implies that either $U_{\alpha}{ }^{\alpha}=0$ and pseudo-Riemannian space has a scalar curvature because of $(2.3)$, or

$$
U_{k}\left(\rho a_{i j}-a_{\alpha i} a_{j}^{\alpha}-\stackrel{1}{\tau} \rho g_{i j}+\stackrel{1}{\tau} a_{i j}\right)+U_{j}\left(\rho a_{i k}-a_{\alpha i} a_{k}^{\alpha}-\stackrel{1}{\tau} \rho g_{i k}+\stackrel{1}{\tau} a_{i k}\right)=0 .
$$

Alternating the latter by indices $j, k$, we will exchange the numeration of indices $i \leftrightarrow k$ and add the resulting expression to (3.4). This will yield

$$
a_{\alpha i} a_{j}^{\alpha}=(\rho+\stackrel{1}{\tau}) a_{i j}-\stackrel{1}{\tau} \rho g_{i j} .
$$

Differentiating this further we obtain

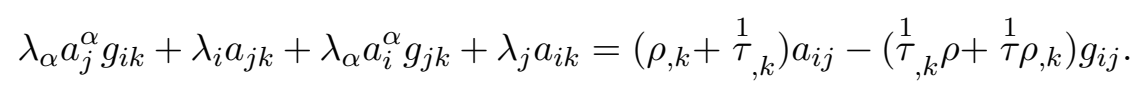

Multiplying (3.6) by $U^{i}$ and contracting it with respect to $i$ we get

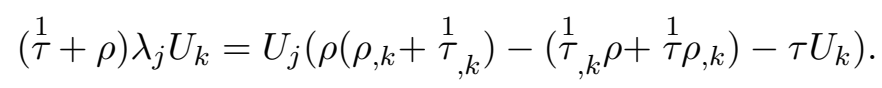

Wrapping it with a vector $\xi^{j}$ chosen in such a way that $U_{\alpha} \xi^{\alpha}=1$, we obtain

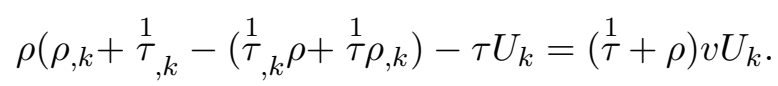

Substituting (3.8) into (3.7) we get

$$
\left(\frac{1}{\tau}+\rho\right) U_{k}\left(\lambda_{j}-v U_{j}\right)=0
$$

The identity $\lambda_{i}=v U_{j}$ leads us to another types of quasi-Einstein spaces which will be treated later. Then we put $\stackrel{1}{\tau}=-\rho$ and the equation (3.5) can be re-written in the following way

$$
a_{\alpha i} a_{j}^{\alpha}=\rho^{2} g_{i j},
$$


and the equation (3.6) can be changed to

$$
\lambda_{\alpha} a_{j}^{\alpha} g_{i k}+\lambda_{i} a_{j k}+\lambda_{\alpha} a_{i}^{\alpha} g_{j k}+\lambda_{j} a_{i k}=2 \rho \rho_{k} g_{i j} .
$$

Contract the latter equation with $g^{i j}$ :

$$
4 \lambda_{\alpha} a_{i}^{\alpha}=n \cdot 2 \rho \rho_{k},
$$

Contracting the same equation with $g^{i k}$ we obtain

$$
(n+2) \lambda_{\alpha} a_{j}^{\alpha}+a \lambda_{j}=2 \rho \rho_{k} .
$$

Multiplying (3.11) by $n$ and subtracting it from (3.12) we obtain

$$
(n(n+2)-4) \lambda_{\alpha} a_{j}^{\alpha}+a n \lambda_{j}=0,
$$

or

$$
\lambda_{\alpha} a_{j}^{\alpha}=\gamma \lambda_{j}
$$

where

$$
\gamma:=-\frac{a n}{n(n+2)-4}
$$

Then (3.11) implies

$$
2 \rho \rho_{k}=\frac{4}{n} \gamma \lambda_{k}
$$

whence (3.10) can be written as follows:

$$
\gamma \lambda_{j} g_{i k}+\lambda_{i} a_{j k}+\gamma \lambda_{i} g_{j k}+\lambda_{j} a_{i k}=\frac{4}{n} \gamma \lambda_{k} g_{i j} .
$$

Multiplying (3.14) by $a_{n}^{k}$ we obtain

$$
\rho^{2}=\gamma^{2}
$$

Then, by multiplying (3.14) by $a_{n}^{i}$, contracting it with $i$, and taking to account (3.9), (3.13) and (3.15), we can transform it into

$$
\lambda_{j} a_{i k}+\lambda_{i} a_{j k}+\gamma \lambda_{i} g_{j k}+\gamma \lambda_{j} g_{i k}=\frac{4}{n} a_{i j} .
$$

Finally subtract (3.16) from (3.14). Then we will get a contradiction because pseudo-Riemannian space admits non-trivial geodesic mappings, namely $a_{i j} \neq c g_{i j}$.

Thus, we have proved the following

Theorem 3.2. Each quasi-Einstein pseudo-Riemannian space of the main type has a constant curvature and its invariant $\mu$ satisfies the following condition

$$
\mu_{, k}=\frac{2 R}{n(n-1)} \lambda_{k}
$$

In next section we will study compact quasi-Einstein spaces "in the large" [1]. 


\section{Geodesic Mappings of COMPACT QUASI-EINSTEIN SPACES OF THE} MAIN TYPE

Consider a Hausdorff space such that for each point there exists neighborhood homeomorphic to a certain domain $R^{n}$. Due to [17], such a space admits a pseudo-Riemannian metric turning it into a pseudo-Riemannian space $V_{n}$. A point $M$ is called a geodesic point of a curve $L$ whenever a tangent vector to $L$ at $M$ satisfies the following condition:

$$
\eta_{, \alpha}^{n} \eta^{\alpha}=\frac{d \eta^{n}}{d t}+\Gamma_{\alpha \beta}^{h} \eta^{\alpha} \eta^{\beta}
$$

A curve consisting of geodesic points only is called a geodesic line belonging to the above-mentioned space. Diffeomorphism that maps every geodesic line $V_{n}$ to another geodesic line $\bar{V}_{n}$, is called a geodesic mapping "in the large".

When geodesics from a certain neighborhood of a point are mapped to a certain neighborhood of another point, then the map is called local geodesic. Evidently, every geodesic mapping "in the large" is also a local geodesic mapping.

The opposite is not true. On the contrary, there are important classes of spaces admitting local geodesic mappings but not admitting mappings "in the large".

Theorems that state non-existence "in the large" of a certain type of spaces are called disappearance theorems, [11]. We will proceed with proof of a disappearance theorem for the compact quasi-Einstein spaces of the main type, starting with the following

Lemma 4.1. Let $V_{n}$ be a pseudo-Riemannian quasi-Einstein space and $\lambda_{i}$ be a vector of constant length. Then the scalar curvature of $V_{n}$ equals to zero.

Proof. Suppose that $\lambda_{i}$ that complies with the (2.14) has a constant length, namely

$$
\lambda_{\alpha} \lambda^{\alpha}=A
$$

where $A$ is a certain constant. By differentiating, we obtain

$$
\lambda_{\alpha} \lambda_{, i}^{\alpha}=0 \text {. }
$$

Taking into account (2.14), it is easy to see that

$$
\mu \lambda_{i}+\frac{R}{n(n-1)} \lambda_{\alpha} a_{i}^{\alpha}=0 .
$$

Covariant derivative of the latter expression after substitution of (1.3), (2.14), and (3.17) can be written as follows:

$$
\frac{3 R}{n(n-1)} \lambda_{i} \lambda_{j}+\left(\mu^{2}+\frac{A R}{n(n-1)}\right) g_{i j}+\frac{2 \mu R}{n(n-1)} a_{i j}+\frac{R^{2}}{n^{2}(n-1)^{2}} a_{\alpha i} a_{j}^{\alpha}=0 .
$$


Multiply the latter equation by $\lambda^{i}$ and contract it with respect to $i$. Then we will get

$$
\frac{4 R A}{n(n-1)}=0
$$

In other words, at least one of the constants $R$ and $A$ should equals zero.

Suppose that a constant $A$ equals 0. Then equations (4.1) can be rewritten as follows:

$$
\frac{3 R}{n(n-1)} \lambda_{i} \lambda_{j}+\mu^{2} g_{i j}+\frac{2 \mu R}{n(n-1)} a_{i j}+\frac{R^{2}}{n^{2}(n-1)^{2}} a_{\alpha i} a_{j}^{\alpha}=0 .
$$

Multiplying (4.2) by $U^{i}$ and contracting it with respect to $i$, we will get

$$
\mu^{2}+\frac{2 \mu R \rho}{n(n-1)}+\left(\frac{R \rho}{n(n-1)}\right)^{2}=0
$$

Differentiating (4.2) and contracting the resulting expression with $U^{i}$, we see that

$$
\frac{2 R}{n(n-1)}\left(1+\frac{2 R \rho}{n(n-1)}\right)=0 .
$$

Suppose $1+\frac{2 R \rho}{n(n-1)}=0$.

According to the above statement and the equation (4.3), $\mu=\frac{1}{2}$. This implies that the scalar curvature vanished.

This proves that in all the cases $R=0$. Lemma is completed.

Let us return back to the issue of geodesic mappings "in the large".

Theorem 4.2. A compact quasi-Einstein space of main type with positive definite metric and positive scalar curvature does not admit non-trivial geodesic mappings "in the large".

O.M. Sinyukova suggested to apply the Hopf-Bochner theorem [16] in a new formulation: if a compact pseudo-Riemannian space $V_{n}$ contains a positive definite invariant quadratic form $G^{\alpha \beta} \eta_{\alpha} \eta_{\beta}$, then for a function $\varphi(x)$ the operator

$$
\Delta \phi=G^{\alpha \beta} \phi, \alpha \beta
$$

does not change a sign, so $\varphi=$ const, and $\Delta \phi=0,[14,15]$. A quasiEinstein space of main type has an invariant

$$
\phi=\lambda_{\alpha} \lambda^{\alpha}
$$

whence

$$
\phi_{i}=2 \lambda_{\alpha, i} \lambda^{\alpha}, \quad \phi_{i, j}=2\left(\lambda_{\alpha i} \lambda_{, j}^{\alpha}+\lambda_{\alpha, i j} \lambda^{\alpha}\right) .
$$

Applying equations (2.14) and (3.17), we can see that

$$
g^{\alpha \beta} \lambda_{i, \alpha \beta}=\frac{(n+3)}{n(n-1)} R \lambda_{i}
$$


Taking this into account, we obtain

$$
\Delta \phi=\frac{2(n+3)}{n(n-1)} R \lambda_{\alpha} \lambda^{\alpha}+2 \lambda_{\alpha, \beta} \lambda_{., .}^{\alpha \beta} .
$$

Since the matrix form $V_{n}$ is positive definite and $R>0$, it follows that $\Delta \phi \geqslant 0$. Then Hopf-Bochner theorem implies that $\phi=$ const, and $\Delta \phi=0$.

Applying Lemma 4.1 we can see that the theorem is proven.

\section{CONCLUSiON}

On studying geodesic mappings of quasi-Einstein spaces with gradient defining vector, it became clear that locally they can be subdivided in three types depending on the existence of solutions of certain equations. Spaces of every type admit non-trivial geodesic mappings.

The "disappearance theorem" is proved for compact quasi-Einstein spaces of main type with additional conditions imposed on metrics and scalar curvature.

\section{REFERENCES}

[1] Alexey V. Bolsinov, Volodymyr Kiosak, Vladimir S. Matveev. A Fubini theorem for pseudo-Riemannian geodesically equivalent metrics. J. Lond. Math. Soc. (2), 80(2):341-356, 2009, doi: 10.1112/jlms/jdp032.

[2] L. E. Evtushik, V. A. Kiosak, I. Mikesh. On the mobility of Riemannian spaces with respect to conformal mappings onto Einstein spaces. Izv. Vyssh. Uchebn. Zaved. Mat., (8):36-41, 2010, doi: 10.3103/S1066369X10080049.

[3] I. Hinterleitner, V. Kiosak. $\varphi$ (ric)-vector fields on conformally flat spaces. Proceedings of American Institute of Physics, 1191:98-103, 2009, doi: 10.1063/1.3275604.

[4] Irena Hinterleitner, Volodymyr A. Kiosak. $\phi(\mathrm{Ric})$-vector fields in Riemannian spaces. Arch. Math. (Brno), 44(5):385-390, 2008.

[5] V. Kiosak, O. Lesechko, O. Savchenko. Mappings of spaces with affine connection. 17th Conference on Applied Mathematics, APLIMAT 2018 - Procedings, pages 563$569,2018$.

[6] V. Kiosak, A. Savchenko, O. Gudyreva. On the conformal mappings of special quasi-einstein spaces. AIP Conference Procedings, 2164(040001), 2019, doi: $10.1063 / 1.5130793$.

[7] V. A. Kiosak, V. S. Matveev, I. Mikesh, I. G. Shandra. On the degree of geodesic mobility of Riemannian metrics. Mat. Zametki, 87(4):628-629, 2010, doi: $10.1134 /$ S0001434610030375.

[8] V.A. Kiosak. On the conformal mappings of quasi-einstein spaces. Journal of Mathematical Sciences, (United States), 184(1):12-18, 2012, doi: $10.1007 /$ s10958-012-0848-6.

[9] Volodymyr Kiosak, Vladimir S. Matveev. There are no conformal Einstein rescalings of complete pseudo-Riemannian Einstein metrics. C. R. Math. Acad. Sci. Paris, 347(1718):1067-1069, 2009, doi: 10.1016/j.crma.2009.06.017.

[10] Volodymyr Kiosak, Vladimir S. Matveev. There exist no 4-dimensional geodesically equivalent metrics with the same stress-energy tensor. J. Geom. Phys., 78:1-11, 2014, doi: $10.1016 / j$.geomphys.2014.01.002. 
[11] Shoshichi Kobayashi. Projective invariant metrics for Einstein spaces. Nagoya Math. $J ., 73: 171-174,1979$, URL.

[12] J. Mikes, I. Hinterleitner, V.A. Kiosak. On the theory of geodesic mappings of einstein spaces and their generalizations. AIP Conference Proceedings, 861:428-435, 2006, doi: $10.1063 / 1 / 2399606$.

[13] N.S. Sinyukov. Geodesic mappings of Riemannian spaces. Nauka, 1979.

[14] E. N. Sinyukova. Geodesic mappings of certain special Riemainnian spaces. Mathematical Notes, 30(6):946-949, 1981.

[15] H. N. Sinyukova. Geodesic uniqueness in the whole of some generally recurrent Riemannian spaces. Journal of Mathematical Sciences, 177(5):710-715, August 2011, doi: $10.1007 / \mathrm{s} 10958-011-0500-\mathrm{x}$.

[16] K. Yano, S. Bochner. Curvature and Betti numbers. Annals of Mathematics Studies, No. 32. Princeton University Press, Princeton, N. J., 1953.

[17] M. Zarichnyi, A. Savchenko, V. Kiosak. Strong topology on the set of persistence diagrams. AIP Conference Procedings, 2164(040006), 2019, doi: 10.1063/1.5130798.

Received: December 16, 2019, accepted: February, 3, 2020.

V. Kiosak

Odesa State Academy of Civil Engineering and Architecture, Didrihson st., 4, ODESA, 65029, UKRAINE.

Email: kiosakv@ukr.net

ORCID: orcid.org/0000-0002-7433-6709

A. Savchenko

Kherson State Agrarian University, Stretenska st., 23, Kherson, 73006, Ukraine.

Email: savchenko.o.g@ukr.net

ORCID: orcid.org/0000-0003-4687-5542

G. Kovalova

Odesa State Academy of Civil Engineering and Architecture, Didrihson st., 4, OdesA, 65029, UKRAINE.

Email: gkovalova@ukr.net

ORCID: orcid.org/0000-0003-2228-2312 